\title{
Editorial
}

\section{Advances in Smart Materials and Applications}

\author{
Kuan Yew Cheong, ${ }^{1}$ Kean Aw, ${ }^{2}$ Khasan S. Karimov, ${ }^{3}$ Feng Zhao, ${ }^{4}$ \\ Mohammad Mahroof-Tahir, ${ }^{5}$ and Rupert Schreiner ${ }^{6}$ \\ ${ }^{1}$ School of Materials \& Mineral Resources Engineering, Universiti Sains Malaysia, Malaysia \\ ${ }^{2}$ Department of Mechanical Engineering, University of Auckland, New Zealand \\ ${ }^{3}$ Faculty of Electronic Engineering of GIK Institute, Pakistan \\ ${ }^{4}$ Department of Electrical Engineering, Washington State University, Vancouver, WA 98686, USA \\ ${ }^{5}$ Department of Chemistry and Earth Sciences, Qatar University, Qatar \\ ${ }^{6}$ Faculty of General Studies \& Microsystems, Regensburg University of Applied Sciences, Germany \\ Correspondence should be addressed to Kuan Yew Cheong; srcheong@usm.my
}

Received 1 April 2014; Accepted 1 April 2014; Published 15 April 2014

Copyright ( 2014 Kuan Yew Cheong et al. This is an open access article distributed under the Creative Commons Attribution License, which permits unrestricted use, distribution, and reproduction in any medium, provided the original work is properly cited.

This is one of a series of special issues published in Advances in Materials Science and Engineering, focusing on the latest advances of smart materials and their applications.

Evolution of engineering materials is strongly depending on the growing transformation of complexity in engineering products. New materials being designed are required to provide specific properties and demonstrate certain functional characteristics by manipulating their dimension, chemistry, and structure through various advanced technologies. Therefore, "smartness" of a material has become the topic of interest. Properties of smart materials may change accordingly to the applied external stimuli.

Under the direction of the editorial team, we showcase advances of organic and inorganic based smart materials and their applications in areas of specific interest such as energy, environment, and health. A total of 9 articles are published in this special issue. Six articles are focused on production, synthesis, and optimization of smart materials; and the remaining are dedicated to application of smart materials.

\section{Acknowledgments}

We would like to express our sincere thanks to all the contributing authors for sharing their research outputs and time, thus enabling the successful accomplishment of this issue.
Khasan S. Karimov Feng Zhao Mohammad Mahroof-Tahir Rupert Schreiner

Kuan Yew Cheong

Kean Aw 

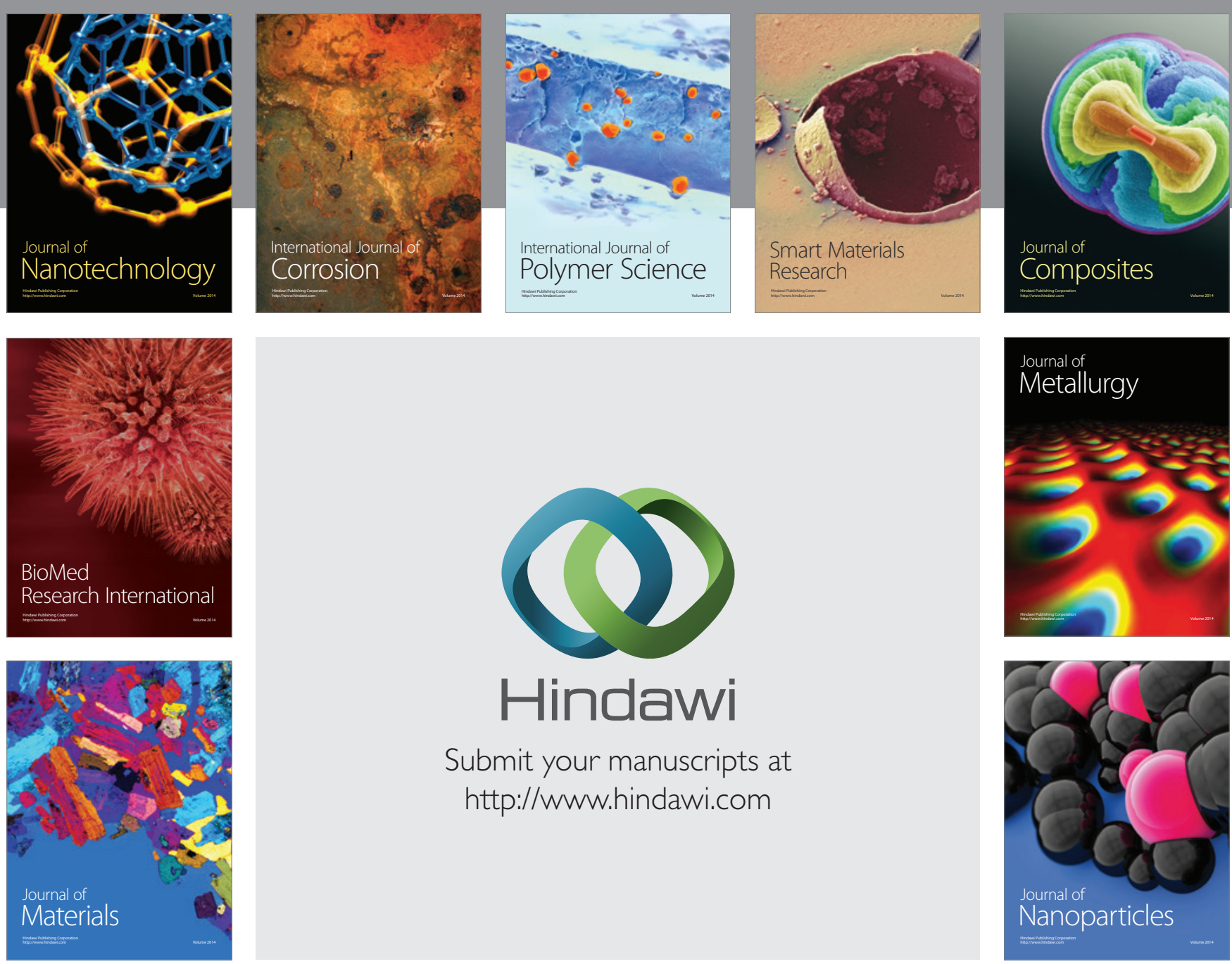

Submit your manuscripts at http://www.hindawi.com
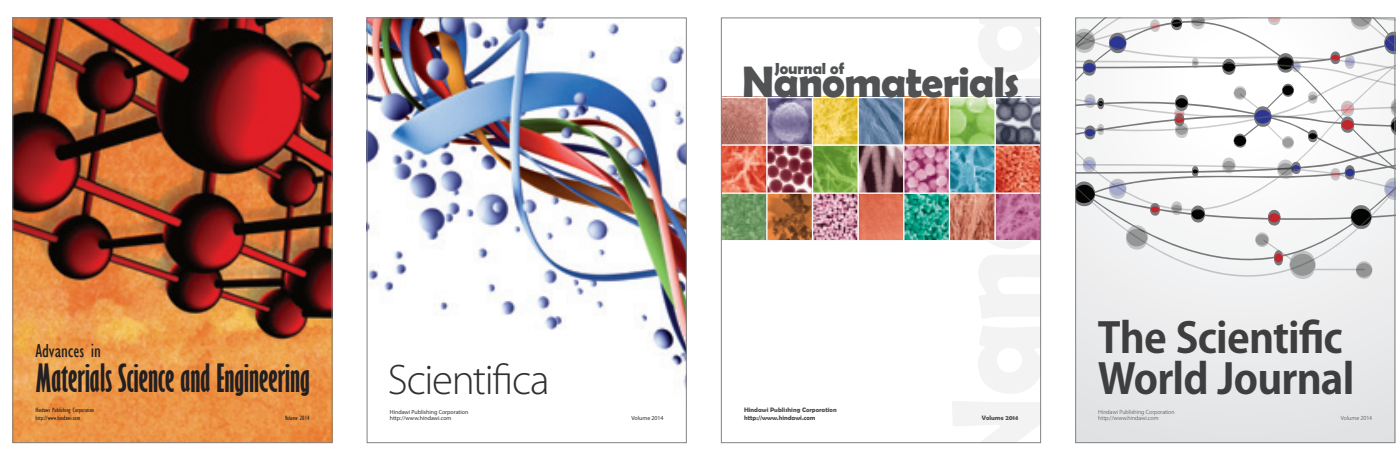

\section{The Scientific World Journal}
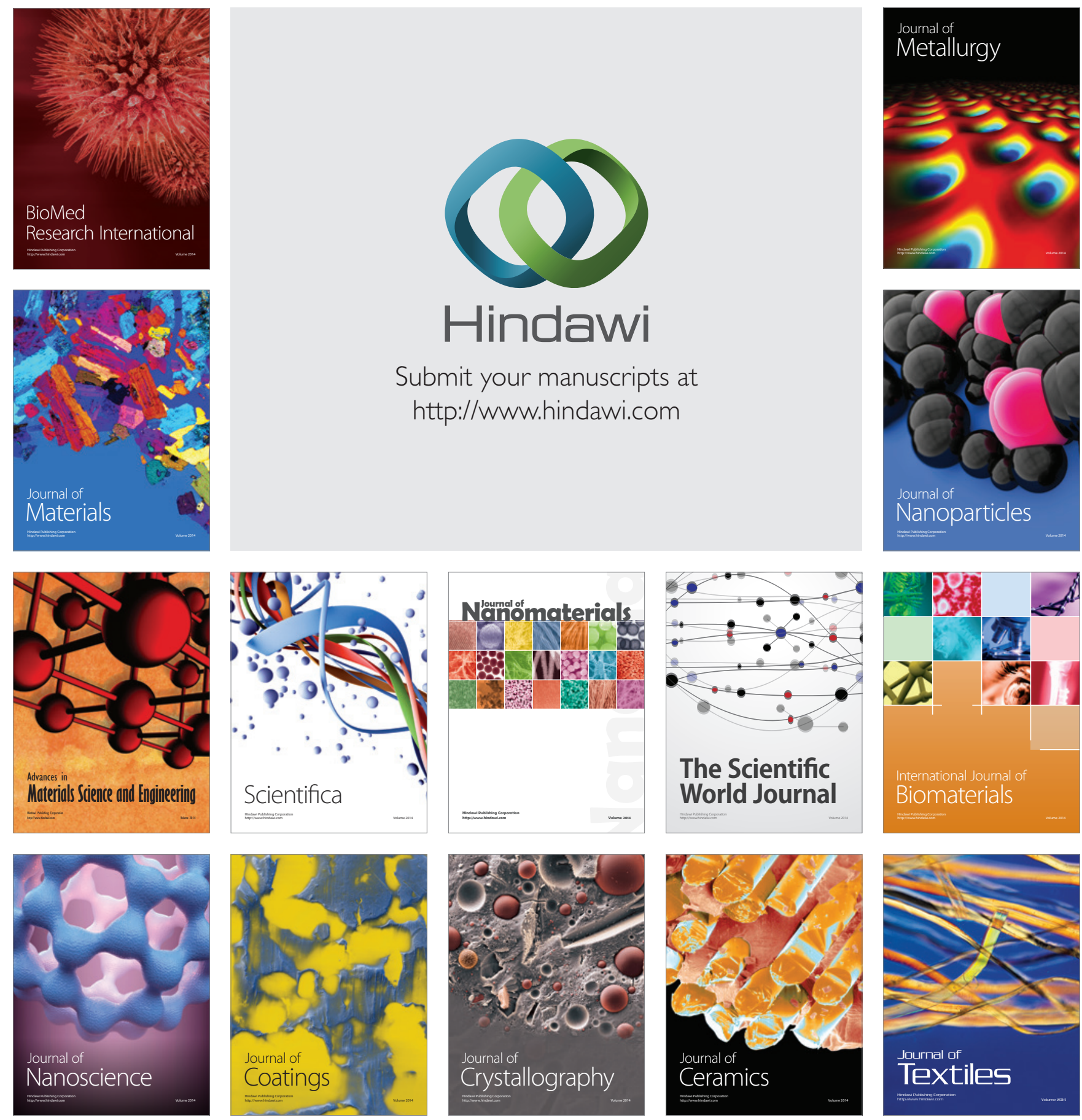